\title{
Experiences of family members caring for Tuberculosis patients at home at Vhembe district of the Limpopo Province
}

\begin{abstract}
Authors:
Joyce T. Sukumani ${ }^{1}$

Rachel T. Lebese ${ }^{1}$

Lunic B. Khoza ${ }^{1}$

Patrone R. Risenga ${ }^{1}$

Affiliations:

${ }^{1}$ Department of Advanced

Nursing, University of Venda

South Africa

Correspondence:

Rachel Lebese

Email:

rachel.lebese@univen.ac.za

Postal address:

Private bag X5050,

Thohoyandou 0950,

South Africa

Dates:

Received: 02 Sep. 2011

Accepted: 28 Feb. 2012

Published: 18 May 2012

How to cite this article:

Sukumani, J.T., Lebese,

RT., Khoza, L.B. \& Risenga,

P.R., 2012, 'Experiences of

family members caring for

tuberculosis patients at

home at Vhembe district

of the Limpopo Province',

Curationis 35(1), Art. \#54,

8 pages. http://dx.doi.

org/10.4102/curationis.

v35i1.54
\end{abstract}

C) 2012. The Authors.

Licensee: AOSIS

OpenJournals. This work

is licensed under the

Creative Commons

Attribution License.
Tuberculosis (TB) is a potentially serious infectious disease which enters the body by inhalation and usually affects the lungs. TB is ranked amongst the leading infectious diseases worldwide, and in South Africa (SA) it has become an epidemic, because of its high prevalence. There are multiple factors that were found to attribute to the existence and spread of this disease. The purpose of this study was to explore and describe experiences of family members caring for TB patients at home, in the Vhembe District of the Limpopo Province. The study was qualitative, explorative, descriptive, phenomenological and contextual in nature. The population group selected for the study all comprised of family members caring for tuberculosis patients at home in Tshifulanani village. A purposive sampling method was applied in the study. Data were collected through semi-structured interviews guided by three questions. Tech's eight steps of data analysis were followed. Measures to ensure trustworthiness and ethical issues were observed. The results of the study revealed that family members experienced difficulties when they care for TB patients at home. These difficulties included providing food, attending to hygiene needs, the lack of equipment, financial constraints as well as physical and psychological exhaustion. Recommendations were made concerning the provision food, attending to hygiene needs, assisting in the administration of medication, nursing practice and policy making.

\section{Introduction}

A family is a group of people who are related to one another either by blood, marriage or adoption and who live together for long periods of time (Du Toit \& Van Staden 2007:143). The father, mother and children form the nuclear family and adults in the family assume the responsibility of caring for the younger members. Some members of the family take care of those who are incapable of caring for themselves because they suffer from TB and other diseases (Du Toit \& Van Staden 2007:143). When a patient receives treatment at home, he or she expects practical support from any member of the family who is responsible enough to take care of the sick. The patient expects emotional support and guidance from these family members and considers home to be the haven for rest and recuperation (Du Toit \& Van Staden 2007:143).

As a caregiver, the family member plays many roles when caring for a TB patient, including the assessment of adverse effects of medication, such as deafness, gastrointestinal upset, skin rashes, hypotension and many other medical problems. In addition, the caregiver should monitor the patient's adherence to his or her medication regimen and should keep a schedule of the patient's medication. The caregiver should also ensure that the patient practices safe hygiene and consumes a nutritious diet. Caregivers should also supervise the patient's levels of activity and ensure that scheduled appointments with primary health care providers are kept (Lewis, Heitkemper \& Dirksen 2005:548; Smeltzer \& Bare 2003:441). These duties and experiences pose challenges and exert pressure on family members who are caring for TB patients at home.

Living with and caring for a TB patient may cause problems within the family that are difficult to deal with. Besides the pain of watching a loved one suffer, family members may experience shame, resentment and guilt. It may be very difficult for family members to accept the illness and adjust to the fact that despite their efforts to care for them, the condition of their loved ones might become worse (South African Depression and anxiety group 2008:5). Members of the family have an important role to play in caring for patients when they are discharged from hospital, and in maintaining the continuity of care required by the Primary Health Care (PHC) approach that has been determined by the South African National Health Plan (ANC 1994:43). The duties of family members may include caring for the patient after early discharge from hospital, providing social support, and carrying out other activities such as leaving for work every morning despite the fact 
that they are also acting as caregivers (Thomas 2002:371). In support of the above statement, Roberfroid (2000:2) indicates that family care practices, coping mechanisms and family care systems have been the foundation of communities for generations.

A study conducted by Shetty, Shemko and Abbas (2004:81) noted that a patient's close family members usually did not ostracise or stigmatise the patient, but they additionally noted that some patients reported offensive treatment by members of the community and their more distant relatives (Shetty et al. 2004:81; Morankar \& Suryawanshi 2000:3; Needham, Bowman, Foster \& Faussett 2004:98). These authors found that family members of TB patient's were ostracised by their communities for staying with the patients. The extent of social stigma on the family may also affect the way in which the family cares for the TB patient. In the Bible, it is told that patients were also isolated in a sanatorium and were kept away from society. Patients were not given emotional, social or physical support by their family members (2 Ki 7:3). In modern times family members with $\mathrm{TB}$ are often stigmatised as being poor because TB is said to be the disease of the poor. Those with TB have been regarded as dirty, as eating bad food and as having bad hygiene (Westway \& Wolmarans 1994:447; Liefooghe, Balidawa, Kipruto, Vermeire \& Demuynck 1997:812).

\section{Problem statement}

At the time of undertaking this research, the Vhembe district had the highest number of TB patients in the Limpopo Province. Most of these patients were treated as outpatients and were cared for by their family members. Some patients were too ill to care for themselves, which meant that family members had to act as carers and supervise the treatment of the patient in their homes. When the researchers accompanied nursing students to the community-based learning projects, the researchers observed that TB patients were discharged from hospitals when they were still critically ill, and in need of further management at home. This occurred even though family members might not have possessed the necessary knowledge and skills to care for the critically ill patients. According to the South African National Health Plan strategy (ANC 1994:143) patients should be admitted to hospital for short periods, of 14 days, and after this they should be discharged to be cared for by their families. However, when these family members have other duties to perform, besides caring for discharged patients, they face many difficulties. Hence, the present study sought to explore the experiences of family members who care for TB patients at home.

\section{Research objectives}

The objectives of this study were to:

- explore the experiences of family members caring for TB patient at home

- identify and describe the caring behaviour of family members when caring for TB patients at home

- identify and describe the challenges faced by family members whilst caring for TB patients at home.

\section{Research method and design Design}

The designs for this study were qualitative, exploratory and descriptive in nature, and the phenomenological approach was followed. The experiences of participants caring for TB patients were explored. The researchers observed, described and later documented lived experiences as related by the participants. The researchers chose the descriptive research method because it summarised the findings of the problem investigated (Burns \& Grove 2001:223; Polit \& Hungler 1991:210; Polit \& Beck 2006:19).

\section{Population and sampling}

The population consisted of all family members from Tshifulanani village in the Vhembe District who were caring for TB patients at home. The researcher involved in site selection applied purposive sampling to select Tshifulanani village in the Thulamela municipality as the site for research, because statistics from one clinic in this area indicated a high prevalence of TB patients. TB patients were selected from the clinic register and traced to their families through a Directly Observed Treatment Strategy (DOTS) supporter. A sample of 13 family members from the village were chosen to participate in the study.

A sample is a subset of the population selected for a particular study (De Vos, Strydom, Fouche, and Delport 2006:193). The sample in this research consisted of only one or two members of each family depending on the size of the family. Males and females were chosen. The inclusion criteria followed were that:

- participants were chosen from families where there was a TB patient on treatment during the time of data collection - participants were family members caring for the patient.

\section{Data collection}

Data were collected using the unstructured interview method. The interviews were conducted as normal conversations with the purpose of producing more in-depth information on the subjects concerned (Brink 1996:158). The unstructured interview is also called a non-scheduled interview. The interviews were prearranged. The researcher established rapport with the participants by paying attention to what they were saying. She did not interrupt the participants, was non-judgemental and developed a healthy appreciation for silence. Discussions proceeded in a relaxed, conversational manner, with questions asked such as:

Can you tell me about your experiences in caring for the patient with TB at home? How can you describe your caring behaviours towards your patients? What challenges do you experience when caring for the TB patient?

The conversations were tape recorded and transcribed verbatim. The researcher also observed the participants for non-verbal cues. The interviews were conducted in the patients' homes. Data collection was terminated when saturation was reached. 


\section{Data analysis}

Data were analysed qualitatively using Tesch's Open Coding method whereby data are broken down into discrete parts, closely examined and compared for similarities and differences. The questions asked were reflected in the data and themes, additionally, categories and sub-categories were developed from the data (Creswell 2003:192).

\section{Results \\ Description of the sample}

As mentioned above, in-depth unstructured interviews were conducted with 13 family caregivers from one village in the Vhembe District. Two of the participants were males aged 38 and 70. One male caregiver was employed as a security officer who had requested to only work night duty in order to care for his wife during the day. The other male caregiver was a pensioner. The rest of the participants were females aged between 35 and 70. Most of the female caregivers worked as domestic servants. Some of them were retrenched after a time owing to their absenteeism from work because they needed to care for a TB patient.

\section{Ethical considerations}

The researcher obtained permission to conduct this study from the following authorities: the Higher Degrees Committee and the Ethics Committee of the University of Venda, and the Chief of the village where the research was conducted. Permission was also obtained from participants through an informed consent form. The recording of interviews, on an audiotape, and the presence of a research assistant were explained to the participants. They were also shown a stop button with which they could stop the recording if they did not wish certain information to be recorded. The consent form was translated into Tshivhenda to assist participants to understand the intentions of the study and their role in it. Participants were also informed about voluntary participation and they were made aware of the fact that they were free to terminate their participation in the study without fear of being victimised. Access to the families was negotiated by the researcher and the research assistant, who took into account the protocol relating to the Vhavenda. This was easily facilitated as the researcher and the research assistant are both from the same cultural group.
Information obtained from the participants was not shared with other people and the recordings were kept under lock and key. Participants were informed about the importance of identifying the challenges that they encountered as they cared for TB patients at home.

\section{Trustworthiness}

Guba's model (in Lincoln and Guba 1985:216) for ensuring and assessing trustworthiness was employed in this study (see Table 1).

\section{Discussion}

Family members described different experiences related to caring for patients with TB at home. Two major themes emerged from the data with categories and subcategories. Appropriate quotes from the raw data and literature substantiate the themes. The themes that emerged are the following:

- challenges related to caring for the TB patient at home

- participant's attitudes related to the carers behaviour.

\section{THEME: Challenges related to caring for a person with TB at home}

Results of the findings indicated the following categories under this theme:

- providing food for the patient

- attending to the patient's hygiene needs

- assisting with the administration of medication.

From the theory of Planned Behaviour, intention was viewed as a determinant of behaviour. In order for family members to undertake their caring role they needed to have the intention to care for a TB patient. Their intention was influenced by their attitudes which could be either positive or negative (Ajzen 1991:180). This is evident in the presented findings.

\section{Providing food for the patient}

The caregivers explained that providing food for the patients involved different aspects, as explained. Six sub-categories emerged from this category which are:

- planting fruit and vegetables

- buying groceries

- cooking for the patient

- feeding the helpless patient

TABLE 1: Strategies used to ensure trustworthiness.

\begin{tabular}{|c|c|c|}
\hline Strategy & Criteria & Application \\
\hline \multirow[t]{4}{*}{ Credibility } & Member checking & $\begin{array}{l}\text { - Data verification with participants to ensure that data were captured properly. } \\
\text { - Use of the audiotape and field notes }\end{array}$ \\
\hline & Prolonged engagement & $\begin{array}{l}\text { - In-depth interviews with follow-up and probing questions to ensure longer contact. } \\
\text { - Rapport established with participants and the use of participants' language ensured extended periods of engagement. }\end{array}$ \\
\hline & Reflexivity & - A field journal was kept throughout the research, reflecting the daily schedule, frustrations, and researcher's thoughts. \\
\hline & Peer review & $\begin{array}{l}\text { - Research process was discussed with supervisor and colleagues. } \\
\text { - Researcher was trained in qualitative data collection methods. }\end{array}$ \\
\hline Transferability & & - Dense description of background information of participants, research context and technique used to select participants. \\
\hline Dependability & $\begin{array}{l}\text { Thick description of } \\
\text { methodology }\end{array}$ & $\begin{array}{l}\text { - Research methods adequately described. } \\
\text { - Code and recoding done. } \\
\text { - Methodology assessed by expects }\end{array}$ \\
\hline Neutrality & Prolonged engagement & - Use of in-depth interviews \\
\hline
\end{tabular}


- cleaning utensils after feeding

- providing information in relation to eating a balanced diet.

Almost all the participants expressed their roles related to caring for the patient as planting fruits and vegetables, buying groceries, cooking for the sick, cleaning utensils after feeding and giving information regarding eating a balanced diet. Some caregivers described their roles as including feeding the helpless patients and encouraging them to eat. The following are some of the quotations from the informants. Not all participants planted fruit and vegetables.

One participant said:

'I always feel tired of caring for the patient because it is now six months and as I told you that I wake up very early in the morning and prepare warm water for the patient to bath and prepare food so that the patient can take medication in a full stomach.' (Participant A)

Rowe et al. (2005:266) and Needham et al. (2004:98) found, in their studies, that family members were expected to provide special food for patients such as eggs, meat, milk, soft drinks, oranges and vegetables which the caregivers could not afford. Rowe et al. (2005:266) concluded that TB patients should not take tablets on an empty stomach. This emphasises the need for them to have food to eat before medication is taken. Chimwaza (2005:104) concurs with this in his study on 'Caregivers experiences with care for HIV and AIDS patients in Malawi'. He stresses the importance of ensuring that patients taking TB and HIV medication have sufficient food to eat because drugs are known to work optimally when a patient consumes them on a full stomach. He goes on to say that medication taken on a full stomach minimises side effects. This means that when insufficient food is taken side effects may occur. These side effects contribute to patients defaulting in their health. Furthermore, most caregivers do not eat nutritious food themselves, although there were some who were assisted by relatives who contributed food to the family (Chimwaza 2005:104). Chanda and Gosnell (2006:6) also indicate the need for good nutrition for TB patients and emphasise the burden this creates for family members caring for TB patients at home. Evans and Thomas (2009:19) found that a lack of food can lead to ill feeling and tension in the family, and this affects the caregiver as he or she is aware that the health and nutrition of the TB patient is important in the healing process.

\section{Attending to personal hygienic needs of the patients}

Almost all care-givers indicated that they were responsible for the hygiene of the patient in one form or another. From the category of being responsible for the hygiene of the patient, the following subcategories emerged:

- assisting with bathing patients who were very ill

- cutting patients' nails and buying their toiletries

- cleaning the patient's environment

- fetching water for the patients who are staying alone

- washing the patient's clothes.
One participant described:

'I used to wake up early in the morning at about 4 am to make fire outside and boil the water so that he can get warm water to bath. He was unable to bath himself and since he is my husband I am the one who was suppose to bathe him and I did not have a choice. Hey!! It was hard I mean like turning him alone to bath his back by the end of the day I was so tired. You know he recovered very fast and now he can bath himself.' (Participant C)

Macq et al. (2005:5) concluded that family members caring for TB patients seemed to have the most harmonious and most supportive behaviour, which was helpful to overcome hardship during illness. Family members are said to motivate the patient to stay healthy and show concern when some members of the family are reluctant to care for patients. This is in contrast with the findings of Bennstam et al. (2004:299) that family members were found to distance themselves from patients suffering from TB and not support the caregivers. The researcher concluded that caregivers experienced hardship when attending to the hygiene needs of patients because they have to turn and bath the patient by themselves, which they found tiring. The caregivers find administering everything for the patient too exhausting, especially when other family members do not assist them. This agrees with the findings of Chimwaza (2005:92) that bathing patients caused caregivers to suffer physical strain, especially when they were alone and they had to assist patients to bathe, turn or go to the toilet. Chimwaza (2005:149) described how caregivers were expected to assist patients to bathe and keep their bodies clean, whilst also cleaning soiled clothes, and bed linen soiled with faeces and menstrual blood. Other caregivers had the challenge of washing without soap because there was no money with which to buy soap.

\section{Attending to environmental hygienic needs of the patients}

The participants described how they sometimes assisted patients to clean their environment and wash their clothing. This was said to be most common when patients were very weak and could not care for themselves. This was described as tiring as caregivers had their own home chores that required their attention. Most of the caring behaviour described by the caregivers included the cleaning of patient's crockery, washing their clothes and cleaning the patient's environment, especially the patient's hut. Water is a basic need for human life, but periodic shortages of water in the villages created further problems. Caregivers described how they had to fetch water for patients for the purposes of washing and bathing. They also complained about the distances from the households to the communal taps. When the taps were dry they were forced to buy water from people with bore holes. This affected both the personal and environmental hygienic needs of the TB patients.

Participant B described how she felt about cleaning the patient's environment especially about having to clean the patient's hut and wash the patient's clothes. She explained:

'When my son was from hospital he was weak and could not do anything for himself so I used to wash and iron his clothes. The 
hut where he was sleeping also needed to be taken care of as he could not do it himself so I also cleaned the environment as well as his hut where he sleeps.' (Participant B)

In most families keeping the patient's clothes and environment clean is the responsibility of the whole family, but in some situations caregivers need to ensure that the environment is clean and free from dust. Ngang et al. (2007:1110) identified that factors contributing to the spread of TB are polluted environments and overcrowding. Caregivers felt the need to clean the environment. Steen and Mazonde (1998:9) concluded in their study that dusty work or mine work was the cause of the disease.

\section{Assisting patients with administration of medication}

The caregivers also described their role as that of assisting patients to take their prescribed medication for treatment. The participants explained the need for patients to take their medication for the recommended period as being important in facilitating their recovery. The caregivers saw their roles in the success of the treatment as being important because they ensured that the taking of the treatment was recorded, and they accompanied patients to the clinic for follow-up treatment. The caregiver administered the medication and recorded and monitored the patient when he or she took the medication. Keeping to this regimen assisted in the patient's recovery. The researcher realised that caregivers were facing many difficulties as they had to know what to monitor, what side effects to look out for, how to record the intake of the medication, and how to adhere to the correct times of administering the medication. Liefooghe et al. (1997:815) described treatments as rough, agonising and even devastating to the patient, hence the need to have family members supervising the patient's taking of the medication.

The following sub-categories emerged from this category, namely:

giving support during the administration of medicine by ensuring compliance with medication regimens:

- DOTS supporters' visits and assistance in the administration of medication

- recording and monitoring the administration of medication on the chart provided

- accompanying the patient to collect medication and equipment

- accompanying the patinet to check-ups.

One participant commented:

'My grandson started taking treatment last year, I wake up early in the morning to prepare soft porridge and take him to the people who help him to take the treatment. I feel good when taking care of my grandson.' (Participant D)

Sagbakken, Frich and Bjune (2008:9) report that there are factors, like the illness of a relative or when the relative needs to attend a funeral, that cause the interruption of TB treatment and which affect compliance. Lack of family support during the administration of medication has also been identified as a major cause of interruption. Sagbakken et al. (2008:9) also indicate that the dynamics of social support mechanisms in the family cause practical changes related to the completion of the first stage of treatment. However, this social support by family members is withdrawn when the patient experiences prolonged bouts of illness.

The present study indicated that social support structures beyond relatives were important in order to ensure compliance with the prescribed treatment. The formation of TB clubs, where members encourage each other to take the full course of treatment, was suggested. Chanda and Gosnell (2006:6) indicated that family members sometimes lose interest in continued supervision and care for the patients. Munthali's findings (in Chanda and Gosnell 2006:6) correspond with those of Sagbakken et al. (2008:9), that the chronic nature of the disease and prolonged treatment protocol can affect compliance to treatment even with family support. It was found that HIV and AIDS is closely related to TB and that some members of the community considered every TB patient to have HIV or AIDS. Family members may condemn TB patients and as a result the patients develop low self esteem and a negative attitude to their condition. Patients may then terminate taking their medication.

\section{DOTS supporter visit and assist in administration of medication}

In a few instances caregivers reported taking the TB patient to a DOTS supporter, but most participants gave medication to the patients themselves. DOTS is a global strategy recognised for the effective control of TB. The DOTS initiative aims to provide all TB patients with the correct support to ensure that they follow the treatment protocol, that being to take their medication at the right time and for the prescribed duration. Anyone from the community, a relative, friend, neighbour or family member can operate as a DOTS supporter who is responsible for giving the patient their treatment and recording the intake on the chart provided. DOTS is considered to be a cost-effective, safe and effective mainstream strategy to control TB (Stop TB country profile 2006; Hane et al. 2007:541).

One participant explained:

'There is a DOT supporter who comes every morning to see to it that the patient takes treatment and record in the book. You see she is helping because I cannot read or write so I would not be able to do what she is doing I think she is doing a great job.' (Participant G)

Travis, Bethea and Winn (2000:412) reported that when family caregivers assisted patients to take their medication problems occurred with giving the medication on time. When family members other than the main caregiver were given the responsibility of monitoring a patient's compliance with treatment protocols, which could include multiple prescriptions, and help the caregiver take his or her own medication, problems sometimes occurred (Travis et al. 2000:412). The administration procedures can also be the cause of the failure of patients to comply with treatment protocols. This is because they find administering certain medications in particular ways extremely taxing. These 
include painful, embarrassing or noxious medication and making decisions at night or during holidays and over weekends, such as whether or not to reduce the dosage or withhold it altogether. In addition they had to monitor safety issues such as the adverse or toxic effects of some medication (Travis et al., 2000:412). Travis et al. (2000:417) also emphasise that the caregiver should remain constantly vigilant. It is important to label prescribed medication and note changes made by the physician to avoid major distress to the caregiver.

\section{THEME: Participant's attitudes related to caring behaviour}

Most participants experienced positive and negative attitudes to their patients. These attitudes affected their caring behaviour. Positive behaviour was experienced when love and sympathy were shown to the caregivers by other members of the family. Five participants indicated this positive behaviour whilst caring for the patient at home. The following are some of the comments made by the informants. One participant said:

'I sometimes feel tired while caring for my child and all of a sudden a telephone call will ring, two children who are away from home always phone me, encouraging me to continue caring for him even if I did not tell them anything. They always tell me that they love me and if it was not for my presence in the family things were going to be bad to their younger brother whom I am caring for. Some relatives keep on paying me a visit and encourage me to continue caring for my child. Most of the relatives show sympathy to me. Some also booked me to the psychologist for counselling as they realised that I am sometimes down. Hmmmm.......I appreciated that and it helped me oh thank God for that.' (Participant A)

Those participants who had negative attitudes towards the patients expressed various feelings. Three indicated that they sometimes had feelings as strong as hatred, whilst others only felt impatience. Feelings of hatred were sparked by the antagonism and hatred shown to them by patients because the caregivers had to ensure that the patients took their medication. One informant described:

'When I start giving him the treatment, he sometimes refuses. He always tells me that he is tired about the many tablets. He will say that he hates me nowadays because of that. I do not care what he says, he is my son I cannot stop giving him the treatment. He is my son. I will take care of him until he is cured. He always shouted saying mummy I hate you, I hate what you are doing to me.' (Participant F)

Participants also experienced feelings of hatred towards their task of caring. This could be the result of the actions of a manipulative patient. Hateful patients can stimulate a series of negative feelings in most caregivers. The participants felt that they were expected to set limits for the patient to limit dependency. James and Groves (1978:887) found that caregivers tended to develop feelings of hatred whilst caring for hateful patients. They also found that there were types of patients who could provoke one to develop hatred towards the act of caring. These patients tended to need continual reassurance, explanations, love and all forms of attention imaginable. Such patients are called 'entitled demanders' because they use intimidation, devaluation and induce guilt, and, thus, they put the carer in the role of an inexhaustible supply depot.

Patients sometimes try to control their carers by using their symptoms as a lever. Others manifest self-destructive behaviour such as excessive drinking. All these types of behaviour affect caregivers adversely and sometimes result in their developing feelings of hatred towards their patients (James and Groves 1978:887). Chimwaza (2005:135) found in his study that caregivers became tired of caring under these circumstances, which led them to become impatient. Sometimes carers projected their feelings of hatred towards thier family members and not just the infected persons. On the other hand some caregivers showed sympathy, indicating that they had positive attitudes towards caring for their TB patients at home. Sympathy is when one is feeling pity for someone else (Chimwaza 2005:131).

Most participants felt that it was very important to have positive attitudes of love towards their patients. One informant said:

'Caring for my mother allows me to show her how much she matters to me. It is my chance to give her something back to her. Sometimes you love someone and do not tell that person. I love my mother. No one can replace her in the whole world. I think I have to spend the time I have with my mother, I have to care for her needs as my mother. I am glad to have been given this chance by God.' (Participant D)

Chimwaza (2005:72) showed that even though participants had to love their patients, they felt that it was their duty to love them and care for them even if they did not wish to perform this role.

\section{Recommendations}

Results from this study also indicate that family members caring for TB patients at home experience financial problems. The problem is especially significant when there is a lack of food. The community is encouraged to have community gardens, gardens at home and community soup kitchens. Amongst the recommendations for the care of TB patients are the following:

- Members of families who are living with TB patients should undergo training before the patient is discharged from hospital. This will enable them to perform their roles and cope with the difficulties they will encounter during the caring period of six to eight months. This will ensure that the patient complies with the treatment regimen and will prevent the adverse effects such as the onset of MDR and XDR.

- Financial constraints should be curbed by the provision of temporary grants for the caregiver to enable him or her to provide for the necessities of the patient, whilst not neglecting other members of the family for whom care is also needed.

- Incentives, such as food, transport fares as well as letters 
of reminder, should be given to caregivers to motivate them to continue to perform their caring tasks.

- Crash courses on nutrition should be provided to caregivers by primary health care nurses at clinics, because most family members seem to lack knowledge of what to buy and how to prepare the required nutritious diet for TB patients.

- The importance of a balanced diet, the consequences of taking treatment before meals, and what to do when a patient does not want to eat, should be part of the crash course.

- More DOTS supporters should be trained in order to have a sufficient number of people to assist caregivers with monitoring, administration of medication and to ensure compliance and, where caregivers are illiterate, to help them record progress.

- There should be DOTS supporters as close to the patients' homes or workplaces as possible. Members of affected families should be encouraged to become DOTS supporters as DOTS supporters that stay far away from the patient might fail to visit the patient on a daily basis.

- The Department of Water Affairs should be consulted to provide taps in areas where there are none and where there is a shortage of water.

- Posts for TB counsellors should be created and they should be stationed at every clinic.

\section{Limitation of the study}

The study was limited to the Vhembe district and it focused on members of families caring for TB patients only. This study was also limited to the Vhavenda people and, as such, cannot be transferable to other cultural groups and districts. Therefore, it is recommended that similar studies are conducted with other cultural groups. The issue of the participant opening up to the researcher was difficult at first, but after they had engaged with the researcher over a period of time, all participants opened up and described their experiences freely.

\section{Conclusion}

Experiences of family members caring for TB patients at home were explored in this study. Family members described their roles and challenges or difficulties that they encountered when they cared for TB patients at home. The study revealed that family members encountered challenges that could affect the completion of treatment by the TB patient. It was discovered that caregivers also have needs that should be met. The roles and difficulties experienced by family members should be urgently addressed in order for the TB patients to receive the best care at home. Family members, as caregivers, need financial, psychological and other types of support as recommended above. They also need education and counselling when caring for patients at home.

\section{Acknowledgement}

South Africa, Netherlands Research Programme on Alternative in Development (SANPAD) for funding the study.

\section{Competing interest}

The authors declare that they have no financial or personal relationship(s) which may have inappropriately influenced them in writing this article.

\section{Author's contributions}

Of the work performed S.T.J. (University of Venda) contributed $40 \%$, R.T.L. (University of Venda) 30\%, L.B.K. (University of Venda) 20\% and PRR 10\%.

\section{References}

Ajzen, I., 1991, The theory of planned behaviour, viewed 09 November 2009, from http://www.valuebasedmanagement, net/methods-ajzen-theory-plannedbehavior.html

ANC Reconstruction and Development Programme, 1994, viewed 25 January 2010 from http://www.africa upenn.edu/Govern-Political/ANC-Reconstruction

Bennstam, A.L., Strandmark, M. \& Diwan, K., 2004, 'Perception of tuberculosis in the DRC (Democratic Republic of Congo: Wali ya Nkumu in the MAI Ndombe district) Qualitative Health Research', Sage Publications, Newbury Park 14(3), 299-312.

Brink, H.I., 1996, Fundamentals of Research Methodology for Health care professionals, Juta and Company Ltd, Kelwyn.

Burns, N. \& Grove, S.K., 2001, Essentials of Nursing Research Practice, W.B Saunders, USA.

Chanda, D. \& Gosnell, D., 2006, 'The impact of tuberculosis on Zambia and the Zambian Nursing Workforce', American Nurses Association 11(1), 61-69.

Chimwaza, A., 2005, 'Caregivers experiences with care for HIV/AIDS patients in Malawi', dissertation, viewed 29 November 2009, from www.malawi.pop.upenn. edu/level\%203/papers/PDF-files/Chimwaza

Creswell, J.W., 2003, Research Design Qualitative, Quantitative, and Mixed Methods Approaches, 2nd edn., Sage Publications, Thousand Oaks.

De Vos, A.S., Strydom, H., Fouche, C.B. \& Delport, C.S.L., 2006., Research at grassroots for Social Sciences and Human Science and Human Service professions, 3rd edn., Van Schaik Publisher, Pretoria.

Du Toit, D.A. \& Van Staden, S.J., 2007, Nursing Sociology, 3rd edn., Butterworth Publishers, Pietermaritzburg.

Evans, R. \& Thomas, F., 2009, 'Emotional interactions and ethics of care caring relations in families affected by HIV and AIDS, Emotions, Space and Society 2(2), 111-119.

Hane, F., Thiam, S., Fall, A.S., Vidal, L., Diop, A.H., Ndier, M., \& Lienhardt, C., 2007 Identifying barriers to effective tuberculosis control. An anthropological approach in Senegal. BMC Health Services Research, viewed 12 January 2011, from http:// www.ncbi.nlm.nih.gov/pmc/articles/PMC (2919530)

James, S.E. \& Groves, M.D., 1978, 'Care of the hateful patient', NEMJ 298(16), 883887. http://dx.doi.org/10.1056/NEJM197804202981605, PMid:634331 The Holy Bible, King James Version. II Kings Chapter 7:3.

Liefooghe, R., Michiels, N., Habib, S., Moran, M.B. \& Demuynck, A., 1997, Perception and social consequences of tuberculosis - a focus group study of tuberculosis patients in Sialkot, Pakistan, Social Science and Medicine 41(12), 812.

Lewis, S., Heitkemper, M. \& Dirksen, S., 2005 'Medical surgical nursing: assessment and management of clinical problems, 7th edn., Mosby, ST. Louis.

Lincoln, Y.S. \& Guba, E.G., 1985, Naturalist enquiry, Sage Publications, Los Angles.

Macq,J.,Solis,A.,Martinez,G.,Martiny,P.\&Dujardin,B.,2005,'Anexplorationofthesocialstigma of tuberculosis in five municipals of Nicaragua to reflect on local interventions, Elsevier: Health Policy 74(2), 205-217. http://dx.doi.org/10.1016/j.healthpol.2005.01.003, PMid:16153480

Morankar, S. \& Suryawanshi, N., 2000, Sociocultural Aspects of Tuberculosis among Women in Western rural Maharashtra, Medicus Mundi Schweiz, India.

Needham, D.M., Bowman, S.D., Foster, S.D., Tomlinson, G. \& Godfrey-Faussett, P., 2002, 'Patient care seeking barriers and tuberculosis programme reform, a qualitative study', Tropical Medicine and International Health 67(1), 93-106.

Ngang, P.N., Ntanganira, J., Kalk, A., Wolters, S., \& Ecks, S., 2007, Perceptions and beliefs about cough and tuberculosis and implications for TB control in rura Rwanda. International Tuberculin Lung disease 11(10), 1108-1113.

Polit, D.F. \& Hungler, B.P., 1991, Nursing Research Method appraisal and utilization, 4 th edn., J.B Lippincot, Philadelphia.

Polit, D.F., Beck, C.T. \& Hungler, B.P., 2006, Essentials of Nursing Research, Methods, Appraisal and Utilization, 6th edn., Lippincott, Philadelphia.

Roberfroid, A., 2000, Stop TB Partnership - Ministerial Conference: Amsterdam 22-24 March.

Rowe, K.A., Makhubele, B., Hargreaves, J.R., Porter, J.D., Hausler, H.P. \& Pronyk, P.M., 2005, 'Adherence to TB preventative therapy for HIV positive patients in rural South Africa implications for antiretroviral delivery in resource poor setting', International journal of Tuberculosis \& Lung Disease 9(3), 263-299. PMid:15786888 
Sagbakken, M., Frich, J.C. \& Bjune, G., 2008, 'Barriers and enablers in the management of tuberculosis treatment in Addis Ababa, Ethiopia: a qualitative study', BMC
Public Health 6(11), 2-14.Shetty, N., Shemko, M. \& Abbas, A., 2004, 'Knowledge, Public Health 6(11), 2-14.Shetty, N., Shemko, M. \& Abbas, A., 2004, 'Knowledge,
attitudes and practices regarding tuberculosis among immigrants of Somalian attitudes and practices regarding tuberculosis among immigrants of Somalian
ethnic origin in London: a cross sectional study, Communicable Disease \& Public ethnic origin in London: a cross section
Health 7(1), 77-82. PMid:15137287

Smeltzer, S.C. \& Bare, B.G., 2003, Textbook of Medical-Surgical Nursing, 9th edn., Lippincott Williams \& Wilkins, Philadelphia.

South African Depression and Anxiety Support Group, 2008, Spectravision Medical Aid Update, 3rd edn.

Steen, T.W. \& Mazonde, G.N., 1998. NGAKA yasetswana, ngaka yasekgoa or both Health seeking behaviour in Botswana with pulmonary tuberculosis, Socia Science 7 Medicine 48(2), 163-172.
Stop TB Country Profile and WHO (World Health Organisation), 2006, Global plan to STOP TB. 2006-2015, viewed 23 February 2011, from http://www.who.int/tb/ features_archive/global_plan_to_stop_tb/en/index.html

Travis, S.S., Bethea, L.S. \& Winn, P., 2000, 'Medication Administration hassles reported by family caregivers of dependant elderly persons', A college of Health Professions University of North Carolina. The Gerontological Society of America 55(7), 417-420.

Thomas, C., 2002, 'Best dissertation section: A literature review of the problems of delayed presentation for treatment and non-completion of treatment for tuberculosis in less developed countries and ways of addressing these problems tuberculosis in less developed countries and ways of addressing these problems
using particular implementation of the DOTS strategy', Journal of Management in Medicine 16(4), 371-400.

Westway, M. \& Wolmarans, L., 1994, 'Cognitive and affective reactions of black urban South Africans towards tuberculosis', Tuberculosis and lung Disease 72(7), 447-453. 